\title{
Radiation Hydrodynamics of the Broad Line Region in Seyfert Galaxies and Quasars
}

\author{
William G. Mathews \\ Lick Observatory and Board of Studies in Astronomy and Astrophysics \\ University of California, Santa Cruz, California 95064
}

\section{Introduction}

The broad line region in quasars and in the nuclei of active galaxies is the site of remarkable hydrodynamic activity unprecedented elsewhere in the universe. Considerable theoretical effort has been directed to determine how this intense radiation is related to high velocity gas motions in these small regions, which, because of their great distances, cannot be resolved by direct observation. A better theoretical understanding of the nature of the broad line-emitting gas involves many novel aspects of radiation hydrodynamics and may eventually provide insights into the nature of the mysterious quasar phenomenon itself.

Continuum and emission line properties of active galaxies and quasars are sufficiently similar that there is little doubt that both can be accounted for by a similar or closely related physical model. The main difference is one of luminosity; typical quasars are considerably brighter than Seyfert galaxies.

In the discussion below the relevant observations of quasars and active galaxies are briefly reviewed with an emphasis on the physical properties of the line-emitting gas and its immediate environment. Arguments that support the importance of radiation forces in producing the observed gas velocities are summarized. Finally, the nature of the acceleration process is described with particular attention paid to the various instabilities that may be present and which are generally characteristic of situations in which plasma velocities result directly from the deposition of radiative momentum. In fact, these troublesome instabilities suggest that radiative forces, although very strong, may provide only a partial explanation of the gasdynamical activity observed.

\section{Observations of the Broad Line Region}

The strongest emission lines observed in ground-based optical observations of active nuclei and quasars are those typical of ionized gas at $T \approx 10^{4} K: \mathrm{C}$ IV $\lambda 1549, \mathrm{C}$ III] $\lambda 1909, \mathrm{~N} \mathrm{~V} \lambda 1240$, Mg II $\lambda 2800$, Ly $\alpha$, and the Balmer lines. Figure 1 illustrates a typical quasar spectrum. A large fraction of the total emission line luminosity is in Fe II radiation (WILLS, NETZER, and WILLS [1]; NETZER [2]). The absence of strong, equally broad forbidden lines of [O II] and [O III] can be understood if plasma densities in the broad line region are sufficiently high, $n \geq 10^{8} \mathrm{~cm}^{-3}$, that these lines are collisionally deexcited. However, densities cannot exceed $\sim 10^{10} \mathrm{~cm}^{-3}$ or observed lines of C III] $\lambda 1909$ and He I would be collisionally deexcited. The impressive widths of these emission lines, $u \approx 5000-20000 \mathrm{~km} \mathrm{~s}^{-1}$, are most often interpreted as evidence for bulk motion of the emitting gas at Mach numbers exceeding 1000! These broad line widths are distinguished from an additional family of lines, the so-called narrow lines, which are typically ten times narrower and, owing to the presence of forbidden lines in this component, are evidently produced in a separate plasma component of somewhat lower density. Although there is some evidence that a connection exists between the kinematics of the broad and narrow 
line regions, as a rule observed line profiles indicate a bimodal distribution of line widths. In any case, the discussion here is limited to the broad line component.

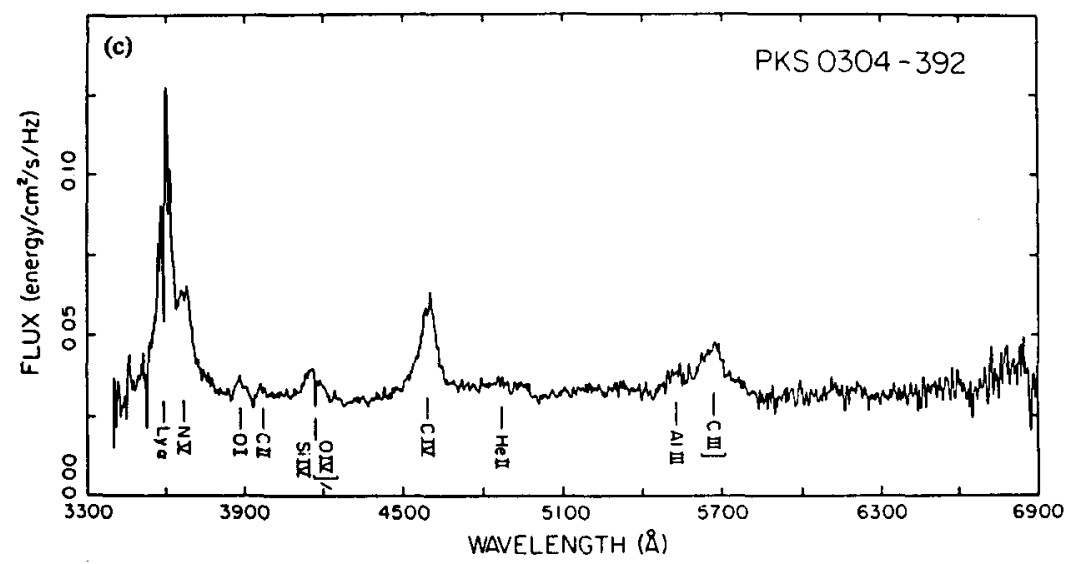

Fig. 1. A spectrum of quasar PKS 0304-392 from Wilkes (1983)

The source of energy that maintains the ionization and thermal equlibrium in the broad line gas is the powerful continuum - a principal defining feature of the quasar phenomenon. The continuum source is evidently considerably smaller than the line-emitting region, as evidenced by the rate of low amplitude continuum variability observed in many sources. The continuum spectrum has often been approximately represented by a power law $F_{\nu} \propto \nu^{-\alpha}$, where $\alpha \approx 1$ from the microwave region to $\sim 1 \mathrm{keV}$, and $\alpha \approx 0.7$ at still higher photon energies. Recently, however, considerable observational evidence has been presented for an additional enhancement in the flux on either side of the UV continuum extending from the Lyman edge to the soft Xray region (SHIELDS [3]; MALKAN and SARGENT [4]; MALKAN [5]). This enhancement not yet fully observed - has been tentatively identified as the thermal continuum produced by an accretion disk orbiting a centrally condensed mass, probably a massive black hole. Owing to uncertainties in the spectrum in the UV bump, which may contain most of the radiatied continuum energy, the bolometric luminosity of the central continuum is uncertain, but is likely to lie in the range $L_{b o l} \sim 10^{45}-10^{47}$ ergs $s^{-1}$ for quasars and approximately 100 times less for Seyfert and broad line radio galaxies.

Many photoionization studies have reproduced the relative emission line strengths (DAVIDSON and NETZER [6]; KWAN and KROLIK [7]; KWAN [8]). Often a single gaseous slab, optically thick in the Lyman continuum, can account fairly well for the relative intensities of the strongest lines, when photoionized from one side by a typical continuum. The expected flux from emission lines can be computed as a function of column depth as shown in Fig. 2. Experience with these calculations indicates that the best line ratios result when the ionization parameter, defined by

$$
\Gamma \equiv \frac{L}{4 \pi R^{2} c} \frac{1}{n}
$$

has a value of approximately 0.01 . $\Gamma$ is the ratio of ionizing photon density to electron density. Adopting a characteristic quasar luminosity $L \approx 10^{46} \mathrm{ergs} \mathrm{s}^{-1}$ and an electron density $n \approx$ $10^{10} \mathrm{~cm}^{-3}$, the value of $\Gamma$ provides an estimate for the scale of the quasar broad line region, 
$R \sim 10^{18} \mathrm{~cm}$. This is considerably larger than the size of the source of continuum radiation inferred from the observed continuum variability. The Strömgren column $N_{s}$ is related to the ionization parameter and the effective recombination coefficient $\alpha$,

$$
N_{s}=c \Gamma / \alpha \sim 10^{22}-10^{21} \mathrm{~cm}^{-2} \text {. }
$$

The physical depth of a line-emitting region, having an optical depth sufficient to produce Fe II and Balmer line emission, is a few times $r_{s}$, where

$$
r_{s} \approx N_{s} / n \sim 10^{11}-10^{12} \mathrm{~cm}
$$

is very much less than $R$. The total mass of emitting broad line gas can be roughly estimated, under Case B conditions, from the observed luminosity in $H \beta, L_{H \beta}$,

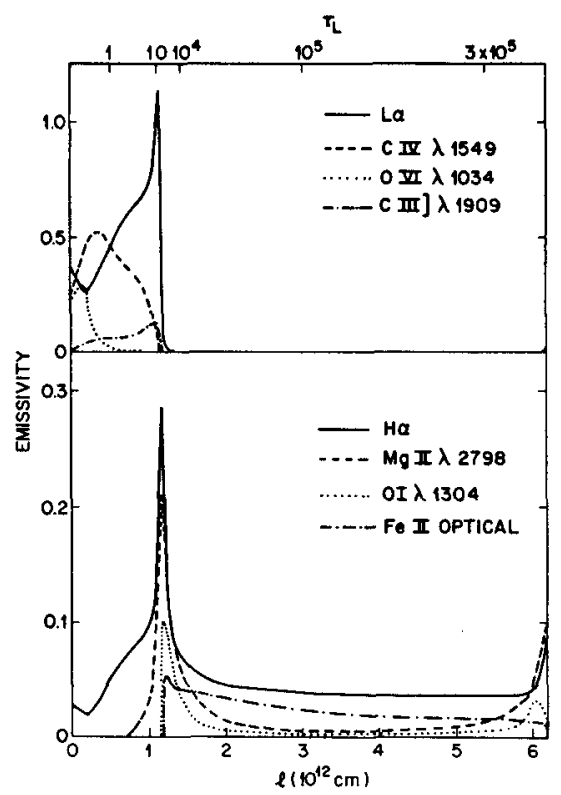

Fig. 2 - Results of a photoionization calculation by KWAN and KROLIK [7] for a slab of nearly solar abundances, density $4 \times 10^{9} \mathrm{~cm}^{-3}, \Gamma=0.03$, and irradiated by a spectrum similar to that of quasar $3 \mathrm{C} 273$. The relative emissivities from prominent highly excited ions (upper diagram) and weakly excited ions (lower diagram) are plotted against physical distance $\ell$ from the irradiated surface, shown at the bottom, and optical depth at the center of Ly $\alpha$, shown at the top. The Strömgren transition is at $\mathrm{r}_{s} \approx 1.2 \times 10^{12} \mathrm{~cm}$. 


$$
M \approx \frac{2}{n_{10}}\left(\frac{L_{H \beta}}{10^{10} L_{\odot}}\right) \quad M_{\odot},
$$

where $n_{10}=n /\left(10^{10} \mathrm{~cm}^{-3}\right)$. Although the total amount of line-emitting gas is insignificant compared to the other massive inhabitants of the central regions of quasars, the mass flux in the broad line gas is not small and represents a puzzling astronomical constraint.

It would be naive to suppose, however, that the quasar emission line spectrum is a superposition of emitting elements, all essentially identical in optical depth, density, and radiative flux to an irradiated plasma slab such as that illustrated in Fig. 2. The principal reason for this is the difference in relative emission line profiles and redshifts observed among lines of different ions. It is well established, for example, that emission lines of more highly ionized lines in quasars show a preferential blue shift relative to the others (GASKELL [9]; WILKES [10]). In addition, MATHEWS and WAMPLER [11] have shown recently that the $\mathrm{Mg}$ II $\lambda 2800$ lines in quasar spectra are systematically narrower than the C IV $\lambda 1500$ lines. Finally, NETZER [2] has remarked on an inconsistancy in the total observed flux in Fe II lines, which can exceed that of Ly $\alpha$, and the total radiative flux incident on slabs such as that shown in Fig. 2 for which $\Gamma \approx 0.01$. These difficulties suggest that the emission line region is spatially stratified and that emission lines arise from regions experiencing a variety of ionization parameters. An even more extreme type of stratification is necessary to account for the observed symmetry in the Ly $\alpha$ line if the emitting elements are regarded as clouds moving radially under the influence of radiation forces. The observed Ly $\alpha$ symmetry would be impossible if all the clouds have radial structures similar to that shown in Fig. 2 for which the optical depth at the center of $\operatorname{Ly} \alpha$ is $10^{6}$ or more. Essentially no Ly $\alpha$ photons could emerge from the nonirradiated side of the clouds and a dramatic emission line asymmetry would result. For models of the emission line region based on radially moving clouds, such as those in which hydrodynamic motions result directly from absorption of radiative momentum, it is necessary to assume that $\mathrm{Ly} \alpha$ radiation arises mostly from a subset of clouds which are optically thin in the Lyman continuum. The emission in Mg II $\lambda 2800$ and Fe II could then be produced by a rather small number of perhaps denser clouds closer to the source of ionizing radiation (cf. COLIN-SOUFFRIN et al. [12]; COLIN-SOUFFRIN, DUMONT, and TULLY [13]; CLAVEL and JOLY [14]). This could quite possibly alleviate the inconsistency in the Fe II flux noted by Netzer. In addition, such optically thick clouds must emit Ly $\alpha / \mathrm{H} \alpha$ ratios significantly below those observed in order to offset the possibly larger $\mathrm{Ly} \alpha / \mathrm{H} \alpha$ ratios generated in the optically thin clouds. Strong Balmer line emission relative to Ly $\alpha$ could be produced if the optically thick clouds have systematically different densities or ionization parameters, have internal dust, or have internal velocity fields sufficient to enhance the escape of Balmer line photons relative to Ly $\alpha$ (MATHEWS, BLUMENTHAL, and GRANDI [15]).

The relatively small equivalent widths of the emission lines in quasars and the absence of absorption at the Lyman edge in quasars of high redshift indicate that more than $90 \%$ of the continuum radiation is unabsorbed by the emission line gas. The fraction of the solid angle subtended by the broad line region as seen from the continuum source - the covering factor $\Omega$ - increases with decreasing luminosity (WU, BOGGESS, and GULL [16]; REICHERT et al. [17]) and may be essentially unity for several low luminostiy Seyferts. In a few of these objects, partial absorption of soft X-rays has been observed, indicating that the central source is covered by clouds of column density $N \sim 10^{22}-10^{23} \mathrm{~cm}^{-2}$ along the line of sight (HOLT et al. [18]; IVES, SANFORD, and PENSTON [19]; REICHART et al. [17]). This is the same column depth required to account for emission lines of $\mathrm{Fe} \mathrm{II}$ and $\mathrm{H} \mathrm{I}$ in the photoionization calculations, 
strongly suggesting that the line-emitting regions are in the form of clouds distributed along the line of sight. Further geometrical information is provided by observations of the Fe K-edge absorption at $7.1 \mathrm{keV}$ and the associated fluorescence line at $6.4 \mathrm{keV}$. Both features are observed with ratios that imply that the absorbing regions, identified with the broad line emitting clouds, are approximately spherically disposed around a relatively small central continuum source. In the absence of evidence to the contrary, it is reasonable to assume that emission lines are produced in an approximately spherical ensemble of clouds in all similar objects, including quasars of high luminosity, where the covering factor is too small to produce observable soft X-ray absorption.

\section{Implications of the Cloud Model for the Broad Line Region}

A comparison of the thermal and gravitational energies in an emission line cloud of size $r \approx$ $10^{12} \mathrm{~cm}$,

$$
\frac{E_{\text {thermal }}}{E_{\text {grav }}}=\frac{3 k T}{M} \frac{3 r}{4 \pi r^{3} n G M}=5 \times 10^{8} T_{4} r_{12}^{-2} n_{10}^{-1},
$$

implies that the line-emitting clouds cannot be self-gravitating. Furthermore, the sound crossing time across the clouds

$$
t_{s c} \approx \frac{r}{c_{s}}=0.02 r_{12} T_{4}^{-1 / 2} \quad y r
$$

is much less than the orbital or flow time of the clouds through the broad line region,

$$
t_{\text {orb }} \approx R / u=30 R_{18} u_{9}^{-1} y r
$$

so a hot, low density medium must be present to confine the clouds.

The rapid motions of the clouds that give rise to the observed broad line profiles can be understood if clouds are either (i) formed at all velocities in the line profile and then slowed by drag forces against the intercloud medium, (ii) confined by a (possibly relativistically) hot low-drag medium and accelerated by gravitational and radiation forces, or (iii) accelerated outward both by a wind in the confining medium as well as by radiative forces. Combinations of these processes are also possible. The last two possibilities, which emphasize the influence of radiation hydrodynamics, are emphasized in the following discussion.

It is commonly supposed that the enormous luminosities of quasars and active galaxies are associated with compact massive objects - probably black holes - of very large mass. For this reason several authors have discussed models in which the broad line clouds are moving in gravitationally bound orbits at velocities implied by the observed line widths, $u \sim u_{9}=$ $u / 10^{9} \mathrm{~cm} \mathrm{~s} \mathrm{~s}^{-1}$. The principal astronomical objection to this type of model is the extremely large masses required,

$$
M_{H}=\frac{u^{2} R}{G}=8 \times 10^{9} u_{9}^{2} R_{18} \quad M_{\odot} .
$$

Such masses would produce orbital motions of $\sim 600 \mathrm{~km} \mathrm{~s}^{-1}$ at $100 \mathrm{pc}$, far in excess of nuclear stellar velocity dispersions so far observed in any galaxy. Furthermore, randomly moving clouds, virally confined by a gravitational field of a central mass of $M_{H 9}=M_{H} / 10^{9} M_{\odot}$, would collide in times

$$
t_{\text {coll }} \approx \frac{t_{\text {orb }}}{6 \pi \Omega}
$$

comparable to the orbiting times

$$
t_{\text {orb }} \approx 500 R_{18}^{3 / 2} M_{H 9}^{-1 / 2} y r
$$


for covering factors $\Omega \approx 0.1$. Collisions among the clouds can be avoided if they are collectively streaming in a regular, nonrandom way through the broad line region. However, all orbiting clouds are strongly sheared by differential Keplerian forces since the clouds are not self-gravitating. Even the radiative ablation provided by differential Poynting-Robertson shear is sufficient to rapidly distort and elongate clouds, with the consequence that they no longer have sufficient optical depth to account for broad emission lines produced by ions in the most optically thick parts of the clouds. Moreover, orbital decay against the confining medium would occur in times comparable to $t_{o r b}$ even if the confining medium is relativistically hot. Detailed estimates of these time scales, as well as a summary of additional difficulties with models of gravitationally confined clouds can be found in MATHEWS $[20,21]$.

If orbiting clouds seem unlikely because of the improbably large central masses required, $M_{H 9} \approx 10$, then radially infalling clouds are unlikely for the same reason. This leaves radial outflow as the most likely kinematics, and there is some direct observational evidence for this possibility. P-Cygni type absorption profiles, incontrovertable indicators of outflow, are associated with the broad emission lines in a subclass of quasars, as shown in the spectrum of PHL 5200 in Fig. 3. In general, whenever absorption features are observed in the emission profiles in Seyfert galaxies, they are invariably on the blue side (WU, BOGGESS, and GULL [16]), further direct evidence for radial outflow. Finally, GASKELL [9] has discovered a systematic blue shift of broad emission lines of ions of high excitation relative to those of low excitation, as if an outflowing system of clouds were partially occulted by an opaque central object or a region of high optical depth to electron scattering.

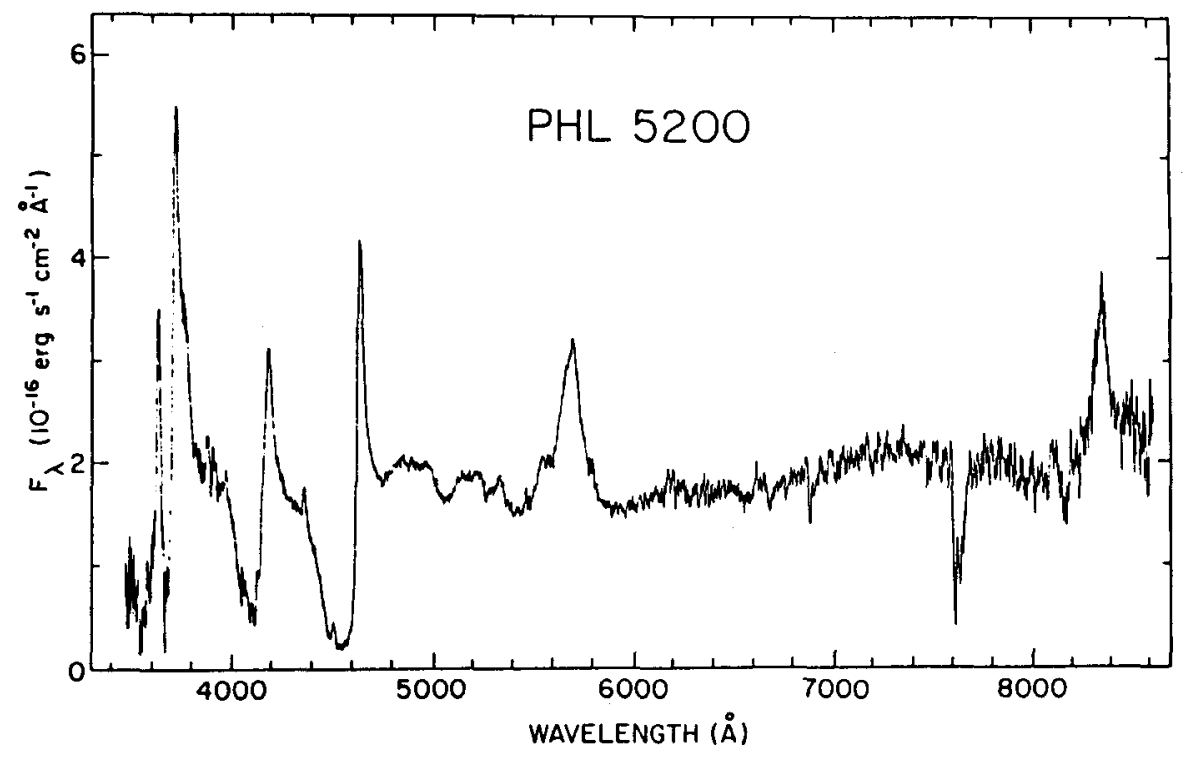

Fig. 3 - A spectrum of the quasar PHL 5200 from JUNKKARINEN, BURBIDGE, and SMITH [22]. Strong blueward absorption troughs, similar to the star P Cygni, accompany many of the prominent emission lines. Ly $\alpha \lambda 1216$ emission redshifted to $\lambda 3630$ is weakened by NV $\lambda 1240$ absorption at $\lambda 3735$. C IV $\lambda 1549$ (at $\lambda 4642$ ) and Si IV $\lambda 1400$ and O IV] $\lambda 1400$ (both at $\lambda 4193$ ) also have blueward displaced absorption bands, but C III] $\lambda 1909$ (at $\lambda 5672$ ) and $\mathrm{Mg}$ II $\lambda 2800$ (at $\lambda 8344$ ) do not. 


\section{Importance of Radiative Forces in the Broad Line Region}

If a flux $F_{\nu}$ of quasar continuum radiation is normally incident on a emission line cloud, the local radiative acceleration at an optical depth $\tau_{\nu}$ from the irradiated surface is

$$
g_{r a d}=\frac{1}{n M} \int \frac{F_{\nu} e^{-\tau_{\nu}}}{h \nu} \frac{h \nu}{c} n \sigma_{\nu} d \nu
$$

where $n$ is the local density at an optical depth $\tau_{\nu}$ from the surface. The relevant opacity $\sigma_{\nu}$ may include absorption in resonance lines, photoionizing absorption in the ultraviolet and $\mathrm{X}$-ray continuum, and electron scattering. A schematic representation of $g_{\text {rad }}$ is shown in Fig. 4. A distinction must be made between $g_{r a d}$, which is a locally defined acceleration, and the integrated acceleration

$$
\left\langle g_{r a d}\right\rangle=\frac{1}{N} \int_{0}^{N} g_{r a d} d N=\frac{1}{N M} \int \frac{F_{\nu}\left(1-e^{-\tau_{\nu}}\right)}{h \nu} \frac{h \nu}{c} d \nu
$$

relevant to the total acceleration of a cloud or plasma slab of column depth $N$.

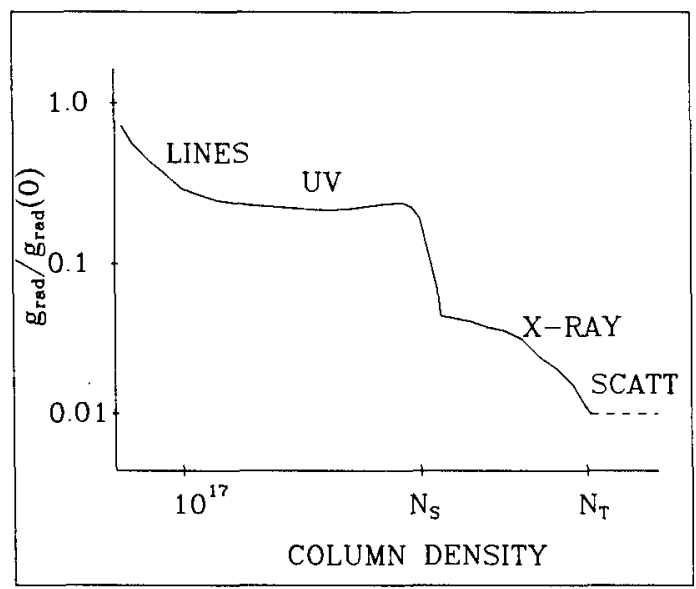

Fig. 4 - A schematic representation of the variation of local radiative acceleration $\mathrm{g}_{\mathrm{rad}}$, normalized to its value at the irradiated boundary $(N=0) . N_{S}$ and $N_{T}$ are the Strömgren and Thompson columns, respectively.

As Fig. 4 indicates, direct absorption of momentum in the resonance lines can exceed that in the continuum only for column depths $N \leq 10^{17} \mathrm{~cm}^{-2}$, much less than the expected size of the broad line clouds. For clouds having $N \approx 10^{17},\left\langle g_{u v}\right\rangle$ exceeds $\left\langle g_{\text {lines }}\right\rangle$. The momentum absorbed in the ultraviolet by photoionization of hydrogen and helium is generated whenever a recombination occurs in the plasma. If the density $n=n /\left(10^{10} \mathrm{~cm}^{-3}\right)$ is roughly constant in the cloud, the ultraviolet acceleration is given approximately by

$$
\left\langle g_{u v}\right\rangle \approx g_{u v} \approx \frac{n \alpha}{M} \frac{\langle h \nu\rangle}{c} \approx 2 n_{10} c m s^{-2},
$$

and is very uniform throughout the cloud. Fully ionized clouds in which the column density $N$ approaches the Strömgren depth $N_{s}$ can experience an enhanced radiative force due to the hardening of the residual radiation (MCKEE and TARTER [23]), but this does not lead to 
disruption of the cloud (BLUMENTHAL and MATHEWS [24], [25]). Clouds having column depths greater than $N_{s}$, can absorb soft X-rays $(h \nu \geq 0.2 \mathrm{keV})$ by K-shell electronic ejection from heavier ions. The complicated variation of the local radiative acceleration in the $\mathrm{X}$-ray region can be approximated quite well with

$$
g_{x} \approx \frac{g_{o}}{\left(1+\frac{N}{N_{o}}\right)}
$$

where $g_{o}$ is proportional to the incident flux in the X-rays and $N_{o}$ is a normalizing column density (MATHEWS [21]). For a $\nu^{-1}$ spectrum the total acceleration by X-rays is comparable to $g_{u v}$. Unlike $g_{u v}$, the local and total acceleration due to X-rays varies inversely with $N$ and this may lead to differential acceleration in clouds having non-uniform column densities (see below). The opacity in the absorption of X-rays varies approximately as $\sigma_{\nu} \propto \nu^{-3}$ and, eventually, as $N$ increases, $\sigma_{\nu}$ falls below the Thompson crossection $\sigma_{T}$. This occurs for photons more energetic than $\sim 8 \mathrm{keV}$. Electron scattering in clouds having columns greater than the Thompson column $N_{T}=1 / \sigma_{T}=1.5 \times 10^{24} \mathrm{~cm}^{-2}$ isotropizes the incident radiation field and reduces the global acceleration. Since all photons having energies between $\sim 8 \mathrm{keV}$ and $1 \mathrm{MeV}$ are absorbed at $N \approx N_{T}$, clouds having initial columns in excess of $N_{T}$ could be truncated by selective radiative acceleration to $N \leq N_{T}$, in agreement with observations of soft X-ray absorption. Finally, the radiative momentum that could be absorbed by dust is small, generally less than $\sim 0.1 g_{u v}$ for reasonable dust-to-gas ratios, in contrast to the situation in galactic $\mathrm{H}$ II regions (MATHEWS [26]). Whether or not dust can exist in broad line clouds has been a controversial issue for some time, but the radiation hydrodymamics may not be strongly affected by this possibility.

The question of central importance is: Can emission line clouds be accelerated to the observed velocities by the action of radiative forces alone, i.e., can $g_{\text {rad }}$ exceed $G M_{H} / R^{2}$ ? For optically thin clouds $N<N_{s}$, radiative acceleration overwhelms gravity for densities

$$
n>7 \times 10^{8} M_{H 9} R_{18}^{-2} \mathrm{~cm}^{-3},
$$

which is easily satisfied by the densities inferred from photoionization studies. If the mass of the central source and stars can be ignored, and if drag forces are not too large, the velocities expected for ultraviolet radiative acceleration

$$
u \approx\left(2 g_{u v} R\right)^{1 / 2}=2 \times 10^{4}\left(n_{10} R_{18}\right)^{1 / 2} \mathrm{~km} \mathrm{~s}^{-1}
$$

are quite consistent with observed line widths. If $\mathrm{R}$ is expressed in terms of the ionization parameter $\Gamma$, the velocity expected from radiative acceleration is a very weak function of the luminosity $\left(u \approx 26000\left(L_{46} n_{10} \Gamma_{-2}\right)^{1 / 4}\right)$, consistent with the similarity of observed line widths in Seyferts and quasars of greatly differing luminosities.

Optically thick clouds having column densities greater than $N_{s}$ can be accelerated outward provided the mass interior to the broad line region does not exceed a critical value $M_{H c r i t}$ defined by

$$
\left\langle g_{\text {rad }}\right\rangle=\left\langle g_{u v}+g_{x}\right\rangle=\frac{G M_{H_{c r i t}}}{R^{2}} .
$$

For a density of $n=10^{10} \mathrm{~cm}^{-3}$, at a distance $R=10^{18} \mathrm{~cm}$ from a quasar of luminosity $L=10^{46}$ ergs $s^{-1}$, detailed calculations (MATHEWS [21]) indicate that the critical masses are $3 \times 10^{9}$ and $1.5 \times 10^{9} M_{\odot}$ for $N=5 N_{s}$ and $N=10 N_{s}$ respectively. The corresponding velocities expected from radiative forces alone $\left(2\left(g_{\mathrm{rad}}\right\rangle R\right)^{1 / 2}$ are 9000 and $6000 \mathrm{~km} \mathrm{~s}^{-1}$ respectively. Since 
reasonable emission line ratios can be produced in clouds having $N \approx 5 N_{s}$, it can be concluded that optically thick, as well as optically thin, clouds can be accelerated to $\sim 10^{4} \mathrm{~km} \mathrm{~s}^{-1}$ by radiative forces alone. However, this can only be done if the drag forces are not excessive and if the clouds remain intact.

Drag forces on radially moving clouds can be neglected provided

$$
N>\hat{n} R,
$$

where variables having circumflexes refer to the intercloud medium. Combining this constraint with the condition for cloud confinement,

$$
n T=\hat{n} \hat{T},
$$

provides a very high lower limit on the temperature of the confining medium:

$$
\hat{T}>\frac{n T R}{N} \approx 10^{10} n_{10} T_{4} R_{18} N_{22}^{-1} \mathrm{~K} .
$$

Unless the gas external to the clouds is essentially relativistic, the forces on the clouds due to their relative motion through the confining medium cannot be ignored. In particular, for the interaction force with the intercloud medium to be ignored, the temperature $\hat{T}$ must significantly exceed the Compton temperature $\sim 10^{8} \mathrm{~K}$ (LEVICH and SUNYAEV [27,28]; MCCRAY [29]; KROLIK, MCKEE, and TARTER (30]; BEGELMAN, MCKEE, and SHIELDS [31]), requiring additional sources of plasma heating.

Cloud acceleration by purely radiative forces can not only generate acceptable emission line widths, but can also produce line profiles in excellent agreement with those observed, although this is a more general property of ensembles of radially moving clouds (BLUMENTHAL and MATHEWS [24]; CAPRIOTTI, FOLTZ, and BYARD [32,33]). Consider a spherically symmetric system of line-emitting clouds, all created at the same initial radius $R_{0}$ at zero velocity and which move out in the quasar atmosphere under the influence of an acceleration $\mathrm{f} / \mathrm{m}$, where the mass in each cloud

$$
m=\frac{4}{3} \pi r^{3} n M
$$

remains constant during its radial trajectory. The equation of motion for the clouds is

$$
m u \frac{d u}{d R}=f
$$

and the equation of continuity for the system of clouds with space density $n_{c}$ is

$$
\dot{m}_{c}=n_{c} m u 4 \pi R^{2} \text {. }
$$

Under these conditions, the shape of one side of the symmetric emission line profile is given by

$$
L_{\nu}=\frac{\dot{m}_{c} c}{2 \nu_{0}} \int_{c\left(\nu-\nu_{0}\right) / \nu_{0}}^{\infty} \frac{\epsilon_{p h}}{(f / m)} \frac{d u}{u} \text { photons } H z^{-1} s^{-1}
$$

Here $\epsilon_{p h}$ represents the emissivity in emission line photons per gram per second, and $\nu_{o}$ is the line rest frequency. 
Whenever the ratio $\epsilon_{p h} /(f / m)$ is essentially constant, the emission line profile is logarithmic, which matches very closely the emission profiles of most unblended lines. For a simple fully ionized cloud accelerated by photoionizing radiation, $f / m=g_{u v} \approx n \alpha\langle h \nu\rangle / M c$ and $\epsilon_{p h}=n \alpha_{\ell} / M$, where $\alpha_{\ell}$ is the effective recombination coefficient for forming a particular line; since both $f / m$ and $\epsilon_{p h}$ depend on density to the first power, a logarithmic line profile results. In addition, this profile is independent of the mass or mass distribution of the fully-ionized clouds and any variation of the confining pressure or shape of the clouds during their radial motion. In the optically thick limit both $f / m$ and $\epsilon_{p h}$ are proportional to $\pi r^{2} F / m$, where $F$ is the radiative flux incident on the cloud, and again a $\log$ profile results. In the case of gravitational infall, $f / m=G M_{H} R^{-2}$, so log-like profiles are possible for optically thin clouds if $n R^{2}$ is reasonably constant over the line-forming parts of the radial orbit. Optically thick clouds falling inward under the influence of gravity can form logarithmic profiles only if $N$ is reasonably constant during their acceleration. Finally, for optically thick clouds being pushed out by a rapidly moving wind in the intercloud medium $(\hat{u}>u)$, then $f / m=\pi r^{2} \hat{\rho} \hat{u}^{2} / m$ and, since $\epsilon_{p h} \approx\left(\pi r^{2} / m\right) L / 4 \pi R^{2}$, a log profile is possible if $\hat{\rho} R^{2}$ and $\hat{u}$ are approximately constant, which is possible for an intercloud wind that has reached terminal velocity (cf. WEYMANN et al. [34]).

For a realistic model of the broad line profiles, it is necessary to specify more accurately the source function for the clouds in the volume surrounding the central continuum source. In particular, it should be recognized that the sum of logarithmic profiles of various widths formed by clouds having various initial radii is not necessary logarithmic.

\section{Radiation-Induced Instabilities}

Even if the radiative forces are sufficient to eject a line-emitting cloud from the quasar atmosphere, a variety of instabilities must be considered which may distort or disrupt the clouds and influence their ability to generate acceptable emission line profiles. Three types of instabilities are considered here: (i) radiative amplification of sound waves, (ii) Rayleigh-Taylor instabilities, and (iii) disruption of clouds having nonuniform column densities.

\section{A. Radiatively Enhanced Sound Waves}

In optically thin clouds or in the fully ionized parts of optically thick clouds, the principal radiative force $g_{u v}$ is proportional to the density. The slight additional condensation in the gas density produced in sound waves can in some circumstances transfer momentum and energy from a nonisotropic radiation field into the waves. This type of instability has been discussed by MATHEWS [35] and MESTEL, MOORE, and PERRY [36] but an earlier version had been developed by HEARN $[37,38]$ for application to radiation-driven hydrodynamic activity in the photospheres of O stars.

To illustrate this instability in its simplest form, consider an idealized isothermal optically thin cloud of uniform density. The radiation force is exactly equal for each part of the cloud, and a coherent uniform acceleration is possible if there are no large dynamical dragging or pushing forces at the cloud boundaries. In this idealized situation, the acceleration of the cloud $a$ is equal but oppositely directed to $g_{u v}$ in the noninertial frame of the cloud. The dispersion relation for sound waves moving in the radial direction is

$$
\omega= \pm k c_{s}(1+i \gamma)
$$


where $\gamma=g_{u v} /\left(k c_{s}^{2}\right)$, and $c_{s}$ is the isothermal sound speed. If $\omega=\omega_{1}+i \omega_{2}$, then

$$
\omega_{2}=\frac{k c_{s} \gamma}{2^{1 / 2}}\left[1+(1+\gamma)^{1 / 2}\right]^{-1 / 2}
$$

is positive for sound waves moving in the same direction as the flux of ionizing radiation. This corresponds to a growing instability such as that illustrated in Fig. 5. Waves moving antiparallel to the radiative flux are damped while those moving transverse to the flux are unaffected. However, unstable waves become nonlinear when the waves have travelled a distance $\sim\left(\omega_{1} / k\right) / \omega_{2}$, which is less than the Strömgren length only if $\Gamma \geq 0.3$. Since this value of the ionization parameter is significantly larger than that required in photoionization studies, most broad line clouds are likely to be stable to radiatively enhanced sound waves.

This instability can nevertheless become important in quasar models involving cool $\left(T \approx 10^{4} \mathrm{~K}\right)$, radiatively-driven winds, where the density gradient helps amplify the instability (MESTEL, MOORE, and PERRY [36]; KIPPENHAHN [39]), or following non-linear density perturbations due to shocks or other disturbances in these extended flows.

\section{B. Rayleigh-Taylor Instabilites}

In the classic Rayleigh-Taylor instability, surface waves at the interface of two incompressible fluids, placed in a uniform gravitational field normal to the surface, obey a dispersion relation

$$
\omega^{2}=-k|g| \frac{\left(\rho_{2}-\rho_{1}\right)}{\left(\rho_{1}+\rho_{2}\right)}
$$

If the density of the upper fluid $\rho_{2}$ is less than that of the lower fluid $\rho_{1}$, stable gravity waves move along the boundary, but if the upper fluid is denser, the equlibrium is Rayleigh-Taylor unstable.

In the noninertial frame of an accelerated quasar cloud, the effective gravity is the difference between the cloud acceleration and the instantaneous local radiative acceleration. Consider a fully ionized cloud, unaffected by dynamical forces at its surface, and which is uniformly accelerated by $g_{u v} \equiv B \rho$, where $B$ is a constant. In this simple case, the instantaneous acceleration field is exactly balanced by the radiative acceleration, the effective gravity vanishes

$$
g_{e f f}=a-B \rho=0,
$$

throughout the cloud and at both surfaces. Therefore $\omega=0$, corresponding to neutral stability.

However, true Rayleigh-Taylor instabilities can occur in more realistic accelerating quasar clouds subjected to the combined influences of radiative and dynamical (i.e. wind) forces. Instabilities may occur at the surface of the cloud - particularly at the surface pushed by a wind - or in the interior of the cloud wherever the density gradient and effective gravity are antiparallel. A reasonable simplified stability criterion for either case is to construct the local effective gravity

$$
g_{\text {eff }} \equiv \frac{1}{M} \frac{d P}{d N}
$$

which can be inserted into a simplified dispersion relation

$$
\omega^{2} \approx-k g_{e f f}
$$



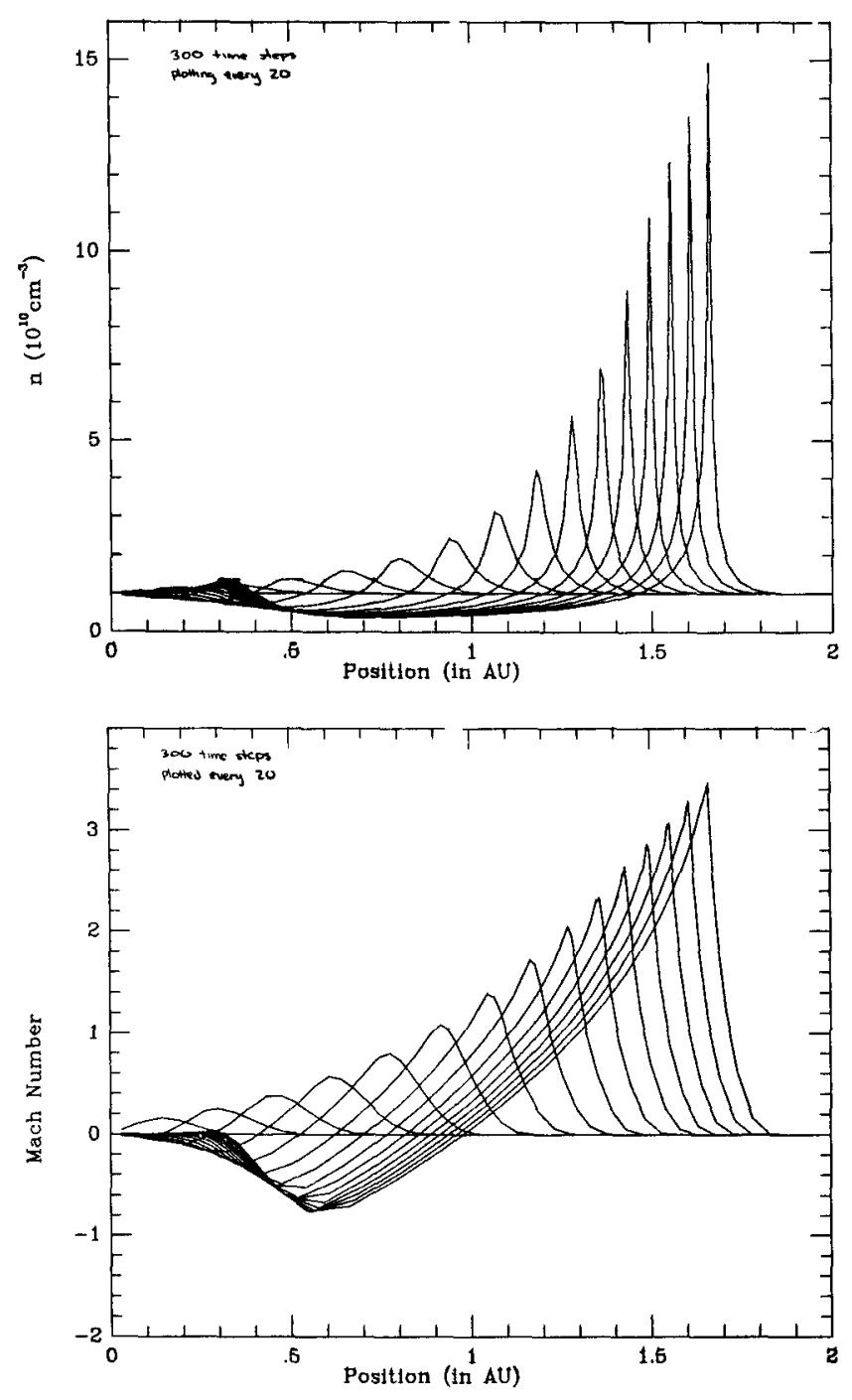

Fig. 5 - Demonstration of the instability of nonlinear, radiatively accelerated waves computed by R. Pogge of Lick Observatory. The initial fully-ionized cloud has a uniform density and is at rest in the noninertial accelerated frame of the cloud. A small, but finite, initial density enhancement exists at the irradiated (left) boundary of the slab. The subsequent development of the density perturbation with time is shown in the upper plot and the corresponding velocity in the lower plot. An outwardly moving shock develops at the leading edge of the nonlinear wave, but this is spread out by artificial viscosity. The rate of progress and growth of the wave follows the linear theory quite well. At the end of the calculation, a second wave is incipient at the left, having been produced from the negative density gradient in the wave of the first wave. 
where the sign of $g_{e f f}$ is negative if it is parallel to the local density gradient either within the cloud or at the surface discontinuities. This obviously underestimates the complexity of the global dispersion relation which must include nonlocal boundary conditions (MATHEWS and BLUMENTHAL [40]) but provides a simple approximate means of determining the R-T instability of any equlibrium configuration.

As an elementary application of this procedure, consider a very optically thin ( $N \ll$ $N_{s}$ ) cloud having a uniform column depth everywhere, such as the one shown in Fig. 6, which is being accelerated by both $g_{u v}$ and a wind in the intercloud medium. The local effective gravity in the noninertial frame of the cloud points from the less dense intercloud medium into the denser cloud at the upper surface in Fig. 6, indicating stability. But the sense of $g_{e f f}$ is opposite at the lower (irradiated) surface, which receives an additional dynamic force from the wind, and this surface is $R-T$ unstable.

These considerations apply only to the linear instability criterion; the nonlinear development may proceed in a quite different manner. The relevant timescale for the nonlinear instability is the freefall time across the cloud in the effective gravity field. Instabilities within the volume of the cloud are often effectively stabilized by the rapid reexpansion of the unstable volume elements to match the ambient pressure (MATHEWS [21]). Surface instabilites, in the nonlinear development, may be partially offset by the enhanced dynamical force on small blobs of cloud gas that fall upstream against the wind, but this is unlikely to restore smooth, unperturbed flow.

The distortion of optically thin clouds by Rayleigh-Taylor instabilities may not drastically influence the emission line profile, since the profile can be quite insensitive to the shape of the cloud. But optically thick clouds cannot be substantially distorted during their acceleration; high optical depths must be maintained to produce broad emission profiles in those lines radiated from optically thick regions. In particular, optically thick clouds having $N>N_{s}$ must be essentially stable to Rayleigh-Taylor perturbations.

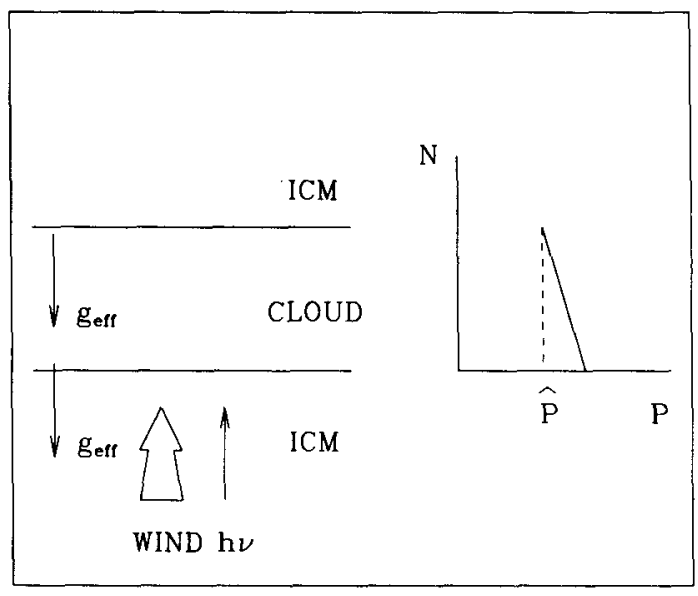

Fig. 6 - A simple model of an optically thin cloud with a uniform column density, surrounded by a hot intercloud medium on both sides. The lower, irradiated surface is pushed by a radial intercloud wind. The distribution of pressure with column depth is shown at the right. $\hat{\mathrm{P}}$, the isotropic ambient pressure in the wind at the position of the cloud, is noted with a dashed line to emphasize the overpressure of the (lower) pushed surface. 
If the energy in the quasar continuous spectrum is reasonably comparable in the ultraviolet and X-ray regions, then the total amount of momentum absorbed by an optically thick cloud in these regions is also comparable. Whenever $N$ is several times larger than $N_{s}$, however, a larger momentum per unit column mass is received in the fully ionized parts of the cloud, and the local acceleration in the most optically thick parts can fall significantly below that of the cloud as a whole. This unequal distribution of acceleration within the cloud has the effect of compressing uniformly accelerated clouds, producing a pressure maximum in their structure, as noted above. If a dynamical pressure, acting at one surface, is not too large, a pressure maximum can occur in this case too. Such a case is roughly illustrated in Fig. 7a, which shows the pressure distribution in a radiatively-accelerated optically thick cloud which experences an additional dynamical pressure at the $N=0$ surface from a wind in the cloud-confining medium. The pressure distribution corresponds to that in the large central region of the cloud which is assummed to have a uniform column depth; the rim regions of the cloud, shown in dashed lines in Fig. 7, do not have the same pressure distribution, as discussed below. Even though the dynamical force at the lower boundary, as indicated in Fig. 7a, is roughly equal in magnitude to the confining pressure, the pressure still has an internal maximum due to the compression by the radiative forces. The local effective gravity points inward everywhere toward the central pressure maximum, so the cloud is probably Rayleigh-Taylor stable everywhere.

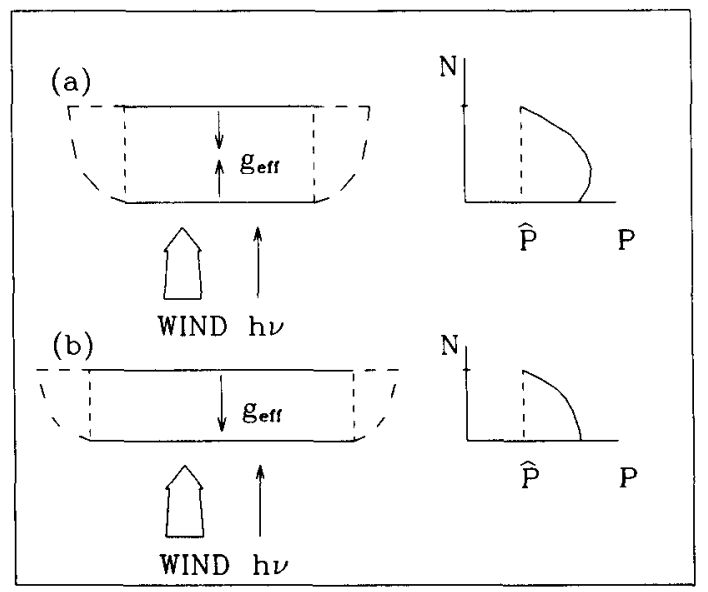

Fig. 7 - Two optically thick clouds pushed by a wind at their irradiated surfaces. (a) shows a cloud in radial hydrostatic equilibrium following an initial transient adjustment. The pressure profile shown at the right has a maximum even though the dynamic pressure of the wind is comparable to $\hat{\mathrm{P}}$. The overpressure within the cloud drives a lateral expansion which should approach a new equilibrium structure shown in (b) where the pressure maximum has moved the pushed surface. The selective acceleration of the rim regions of these clouds, however, may disrupt the equilibrium structures.

Unfortunately, the cloud structure shown in Fig. 7a can be in quasi-hydrostatic equilibrium only in the radial direction since the overpressure at the maximum is greater than the sum of the confining isotropic and dynamical pressures. As a result of this overpressure, the cloud shown in Fig. 7a will expand in a direction transverse to the direction defined by the wind and the radiative flux. Following this transient lateral expansion, which takes approximately one lateral sound crossing time, the radial structure of the cloud resembles that shown in Fig. $7 \mathrm{~b}$, in which the pressure maximum has migrated to the surface that receives the additional 
dynamic pressure. If the dynamical pressure is not too large, the lateral expansion stops when the effective gravity at the surface confronting the wind vanishes, $g_{\text {eff }}=(d P / d N) / M \approx 0$ at $N=0$, the pancake condition discussed by BLUMENTHAL and MATHEWS [24]. The lower surface in Fig. 7b is therefore neutrally stable. This simple example, one of several discussed in more detail by MATHEWS [21], suggests that Rayleigh-Taylor stability is promoted by the presence of radiative forces within optically thick clouds, even when they are pushed by winds as strong as the confining pressure itself. For sufficiently large dynamical pressure, however, the effective gravity at the pushed surface becomes negative and an R-T instability must occur.

Transient overpressures and associated transverse flow can also occur in fully-ionized clouds which are somewhat thicker than the very optically thin cloud considered earlier and shown in Fig. 6, since in this case too the radiative acceleration is no longer exactly uniform in the cloud owing to the additional contribution of $g_{x}$ to $g_{\mathrm{rad}}$. Again, the compressive influence of differential radiative forces is $\mathrm{R}-\mathrm{T}$ stabilizing.

Throughout this discussion illustrating how initial radial hydrostatic equilibrium is achieved and how it may evolve into a new equilibrium configuration following a lateral flow, we have implicitly assumed that the peripheral parts of the clouds can accommodate the combined radiative and dynamical forces. In the following section we show that this is unlikely to be the case.

Before leaving this discussion of the Rayleigh-Taylor instability, another related instability resulting from gradients in the Ly $\alpha$ pressure must be discussed. If there is no dust within the fully ionized parts of quasar clouds, a debatable point perhaps, the isotropic pressure in Ly $\alpha$ photons, $P_{\alpha}$, can approach $10 \%$ of the plasma pressure (see MATHEWS [25] for a simple estimate of this ratio). This does not affect the pressure profiles shown in Fig. 7, however, provided the pressure there is interpreted as the combined pressure of $\operatorname{Ly} \alpha$ and plasma. However, gradients in the resonance line photon pressure can exceed the local effective gravity, i.e.,

$$
\frac{1}{M}\left|\frac{d P_{\alpha}}{d N}\right|>g_{r a d}-a \equiv g_{e f f}
$$

where $a$ is the cloud acceleration. The consequences of this may have serious implications.

The structure of quasar clouds in the presence of $P_{\alpha}$ has been studied by WEYMANN [41] and WILLIAMS [42], and a detailed stability analysis has been presented by KROLIK [43,44]. As a basis for his linear perturbation analysis, Krolik considers an optically thin, isothermal, incompressible finite slab in which a modest fraction of the total pressure is in Ly $\alpha$ radiation. The slab is assumed to be uniformly accelerated by $g_{u v}$ with no additional dynamical forces at its boundaries. Even though the total pressure in such a slab is uniform, the Ly $\alpha$ pressure $P_{\alpha}$ must always have a maximum near the central plane of the slab (or slightly displaced toward the irradiated side due to the nonuniform distribution of neutral hydrogen atoms), and the gas pressure must have a corresponding minimum. However, in order to make a very complex analysis tractable, Krolik assumed that gradients in the gas density were neglible. i.e., that the density scale height is much greater than the wavelength of the perturbation and the mean free path of Ly $\alpha$ photons at line center.

With this zero order structure, linear gravity waves were imposed throughout the slab. The resulting dispersion relation had the form $\omega^{2}=-k|a| A$, where $a$ is the cloud acceleration and $A$ is a positive constant of order unity. This indicates that the fully ionized slab is almost as Rayleigh-Taylor unstable as it would be in a pure gravitational field of magnitude $a$, uncompensated by the influence of $g_{u v}$ ! 
The precise origin of this instability is somewhat obscure. It seems to derive from the anisotropy of the perturbed component of the Ly $\alpha$ pressure tensor, and in this sense the instability may differ from other types of Rayleigh-Taylor instabilities. It is likely that the internal flow of $\operatorname{Ly} \alpha$ photons is responsible for the instability found by Krolik. For example, imagine a fully ionized slab in which an unstable perturbation is in progress. Non-traveling unstable gravity waves correspond to adjacent rising and falling regions within each wavelength of the perturbation. Ly $\alpha$ photons produced in one of these regions, if preferentially absorbed in the receding part of the adjacent counterstreaming regions, could provide a mechanism for the anisotropic Ly $\alpha$ radiation to drive the unstable motion already assumed to be in progress. However, except for second order boundary effects, the additional Doppler absorption available to line photons moving into adjacent counterstreaming regions would, by symmetry, be the same for approaching or receding parts of the adjacent flow - so the counterstreaming would not be sustained by the absorbed radiative momentum. In any case, this unusual instability discovered by Krolik should be studied further and extended to more general cases involving optically thick clouds.

\section{Derimming Instabilities}

Provided quasar clouds are reasonably stable to Rayleigh-Taylor instabilities, and there is certainly no guarantee of this, two additional considerations may undermine the coherence of optically thick clouds: (i) the inability of less optically thick parts of these clouds to be in complete quasi-hydrostatic equlibrium with the rest of the cloud and, (ii) the differential acceleration within clouds having nonuniform column depths.

Consider, for example, a cloud similar to the idealized clouds shown in Fig. 7. This axisymmetric cloud is assumed to have a central cylindrical section surrounded by narrower rims required to balance the additional dynamical (ram) pressure of the wind and allow the total pressure along the rims to balance the pressure in the main body of the cloud. Unfortunately, this arrangement does not fully accommodate the lateral pressure structure. It is possible, of course, to find one dimensional (radial) pressure profiles through any column in the cloud as if it were a part of an infinite slab having a uniform column density equal to that part of the cloud. But in practice when this is done, the radial pressure profiles in the rims do not exactly match that in the main body of the cloud and a lateral pressure gradient must develop. This arises from the unique dependence of $g_{\text {rad }}(N)$ on $N$ in optically thick clouds. Therefore, it is not possible to find a fully self-consistent quasi-static model cloud in the optically thick limit realistic clouds of this type must develop internal circulation.

The second difficulty is even more serious. If a cloud such as those shown in Fig. 7 were to exist momentarily with a low bulk radial velocity, its rims would be accelerated faster than the rest of the cloud, i. e., $\left.\left\langle g_{\text {rim }}\right\rangle\right\rangle\left\langle g_{b o d y}\right\rangle$ since $N_{\text {rim }}<N_{b o d y}$. This selective acceleration can occur for two reasons. First, if the dynamical pressure of the wind dominates the acceleration of the cloud, then the inertia per unit area varies as $N^{-1}$ so the wind will accelerate the rims away from the rest of the cloud. Secondly, this same differential acceleration can occur for any optically thick cloud accelerated in part by X-rays for which $\left\langle g_{x}\right\rangle \propto N^{-1}$, resulting in a negative $d\left\langle g_{\text {rad }}\right\rangle / d N$. In either case, as soon as the rims are removed the unbalanced transverse pressure gradient in the main body of the cloud, generated by the combined dynamical surface force of the wind and $g_{\text {rad }}$, pushes gas laterally from the main body of the cloud in an attempt to form new rims. After only a few lateral sound crossing times, the entire mass of the optically thick cloud will have been processed through this rim flow, the mean optical depth will have been reduced, and the ability of the cloud to radiate broad emission lines from the optically thick 
regions is doubtful.

The best way out of this difficulty is to assume that optically thick clouds are accelerated primarily by a wind in the intercloud medium and that the full velocities required by the broad line widths are reached in just a few (lateral) sound crossing times. The Rayleigh-Taylor stability of the cloud can be enhanced during this acceleration by the compressive internal radiative forces which must always be present.

\section{Conclusions}

In this review, the focus has been on the role of radiation hydrodynamics in accelerating quasar clouds that are assumed to form at relatively low velocities by some unknown process in the broad line region. This is a useful inquiry since radial cloud motion is the kinematical arrangement preferred by observations and general theoretical considerations. More evidence exists for radial motion in the outward, rather than the inward, sense, but this evidence may not be compelling in every case.

There is little doubt that radiation is a powerful force in accelerating line-emitting clouds, but additional dynamical forces are negligible only if the temperature of the confining medium is relativistic. Optically thin clouds may suffer from Rayleigh-Taylor instabilites (if very optically thin) or from the Ly $\alpha$-driven instability, but can still be driven radially out of the quasar atmosphere by either $g_{\text {rad }}$ or $g_{\text {wind }}$. Optically thick clouds, however, are derimmed before significant radial acceleration can occur if $g_{\text {rad }}$ dominates the acceleration. This fate can be avoided if optically thick clouds - and probably optically thin ones as well - are accelerated outward primarily by the dynamical action of a wind. Such clouds may be Rayleigh-Taylor stabilized by the additonal influence of internal radiative forces during thier brief periods of rapid acceleration.

Many of the insights into the nature of the radiative acceleration in optically thick clouds have been possible only with the aid of an elaborate photoionization code generously made available by Gary Ferland, who must receive a special thanks here. The National Science Foundation generously supported this study through grant AST 83-12971.

\section{REFERENCES}

1. Wills, B. J., Netzer, H., and Wills, D. 1985, Ap. J., 288, 94.

2. Netzer, H. 1985, Ap. J., 289, 451.

3. Shields, G. A. 1978, Nature, 272, 706.

4. Malkan, M. A., and Sargent, W. L. W. 1982, Ap. J., 254, 22.

5. Malkan, M. A. 1983, Ap. J. (Letters), 264, L1.

6. Davidson, K., and Netzer, H. 1979, Rev. Mod. Phys., 51, 715.

7. Kwan, J., and Krolik, J. H. 1981, Ap. J., 250, 478. 
8. Kwan, J. 1984, Ap. J., $283,70$.

9. Gaskell, C. M. 1982, Ap. J., 263, 79.

10. Wilkes, B. J. 1984, Mon. Not. Roy. Astron. Soc., 207, 73.

11. Mathews, W. G., and Wampler, E. J. 1985, Publ. Astron. Soc. Pac., in press.

12. Colin-Souffrin, S., Dumont, S., Heidmann, N., and Joly, M. 1980, Astron. Ap., 83, 190.

13. Colin-Souffrin, S., Dumont, S., and Tully, J. 1982, Astron. Ap., 106, 362.

14. Clavel, J., and Joly, M. 1984, Astr. Ap., 131, 87.

15. Mathews, W. G., Blumenthal, G. R., and Grandi, S. A. 1980, Ap. J., 235, 971.

16. Wu, C.-C., Boggess, A., and Gull, T. R. 1983, Ap. J., 266, 28.

17. Reichert, G. A., Mushotzky, R. F., Petre, R., and Holt, S. S. 1985, Ap. J., in press.

18. Holt, S. S., Mushotzky, R. F., Becker, R. H., Boldt, E. A., Serlemitsos, P. J., Szymkowiak, A. E., and White, N. E. 1980, Ap. J. (Letters), 241, L13.

19. Ives, J. C., Sanford, P. W., and Penston, M. V. 1976, Ap. J., (Letters), 207, L159.

20. Mathews, W. G. 1967, Ap. J., 258, 425.

21. . 1985, Ap. J., submitted.

22. Junkkarinen, V. T., Burbidge, E. M., and Smith, H. E. 1983, Ap. J., 265, 51.

23. McKee, C. F., and Tarter, C. B. 1975, Ap. J., 202, 306.

24. Blumenthal, G. B., and Mathews, W. G. 1975, Ap. J., 198, 517.

25. . 1979, Ap. J., 233, 479.

26. Mathews, W. G. 1967, Ap. J., 147, 965.

27. Levich, E. V., and Sunyaev, R. A. 1970, Astroph. Letters, 7, 69.

28. . 1971, Sov. Astron. Jour., 15, 363.

29. McCray, R. A. 1979, in Active Galactic Nuclei ed. C. R. Hazard and S. A. Mitton (Cambridge: Cambridge University Press).

30. Krolik, J. H., McKee, C.F. and Tartar, C.B. 1981, Ap. J., 249, 422.

31. Begelman, M. C., McKee, C. F., and Shields, G. A. 1983, Ap. J., 27170.

32. Capriotti, E., Foltz, C., and Byard, P. 1980, Ap. J., 241, 903.

33. . 1981, Ap. J., 245, 396.

34. Weymann, R. J., Scott, J. S., Schiano, A. V. R., and Christiansen, W. A. 1980, it Ap. J., $262,497$.

35. Mathews, W. G. 1976, Ap. J., 207, 351.

36. Mestel, L., Moore, D. W., and Perry, J. J. 1976, Astron. Ap., 52, 203.

37. Hearn, A. G. 1972, Astr Ap., 19, 417.

38. . 1973, Astr. Ap., 23, 97.

39. Kippenhahn, R. 1977, Astr. Ap., 55, 125.

40. Mathews, W. G., and Blumenthal, G. R. 1977, Ap. J., 214, 10. 
41. Weymann, R. J. 1976, Ap. J., 208, 286.

42. Williams, R. E. 1972, Ap. J., 178, 105.

43. Krolik, J. H. 1977, Phys. Fluids, 20, 264.

44. . 1979, Ap. J., 228, 13.

45. Eilek, J.A. and Caroff, L.J. 1979, Ap. J., 233, 463.

46. Mathews, W.G. 1983, Ap. J., 272, 390.

47. Verbunt, F., Fabina, A.C., and Rees, M.J. 1984, Nature, 309, 331.

48. Ferland, G.J. and Elitzur, M. 1984, Ap. J. (Letters), 285, L11.

49. Shields, G.A., and McKee, C.F. 1981, Ap. J. (Letters), 246, L57. 


\section{QUESTIONS} ionization?

P. Noerdlinger: From the spectroscopy, what limits can you set on cosmic ray type

Mathews: For the broad line clouds, even an otherwise tiny magnetic field can mirror charged cosmic rays at the surface. Protons of energy $\gamma_{4} \equiv 10^{-4} E / M c^{2}$ reflect at a depth $r_{L} \approx 2 \times 10^{11} \gamma_{4} \beta^{1 / 2} n_{10}^{-1 / 2} \mathrm{~cm}$ from the surface of the cloud, where $\beta$ is the ratio of gas to magnetic pressure inside the cloud. One hopes for $\beta<1$ since a large magnetic pressure would give rise to internal density gradients in the clouds and result in disruptive differential acceleration. Obviously, if the energy of the cosmic rays were sufficiently large, a cloud could be penetrated by them, but it is easy to show that particles that completely penetrate the clouds cannot be important sources of cloud heating or ionization. To be effective heaters, the cosmic ray particles must have stopping lengths comparable to the cloud size, which selects out only a relatively small fraction of the total cosmic ray energy. See BLUMENTHAL and MATHEWS [24] for more details.

George Fisher: One aspect of the emission line clouds you didn't discuss was heating of the clouds by the hot $\left(T \sim 10^{10} K\right)$ intercloud medium. A back-of-the-envelope calculation of the stopping depth of a $10^{10} \mathrm{~K}$ electron is $\sim 10^{23} \mathrm{~cm}^{-2}$. This is comparable to (I think) the Strömgren length. Wouldn't this heat up the cloud a lot and evaporate it? Another way of looking at the problem is in terms of a classical conductive flux. This would tend to evaporate the clouds. Finally, since there are all of these mechanisms [that] dissipate clouds, where do they come from?

Mathews: Beware of a back-of-the-envelope calculation on this problem! The principal concern is again the small magnetic field that must exist inside these line-emitting clouds. (If no magnetic field were present in the clouds, it would be the most astonishing attribute of quasars and active galaxies!) When a magnetic field is present, for all practical purposes, thermal conductivity is possible only along the field since the transverse conductivity is many, many orders of magnitude smaller. The efficiency of cloud evaporation then depends critically on whether or not the field within the cloud is connected to the ambient field in the cloudconfining hot gas. If it is assumed that the cloud is moving relative to the ambient medium, however, the field can connect only if the relative velocity is less than some fraction $(\sim 0.1)$ of the Alfven speed in the hot gas. It can be shown that field reconnection is unlikely or impossible if the relative cloud-intercloud velocity exceeds $200 \hat{\beta}^{1 / 2} T_{8}^{1 / 2} \mathrm{~km} \mathrm{~s}^{-1}$. Since the absolute cloud velocities are much larger than this, the velocity of the cloud relative to its local environment is also likely to be large, suggesting that internal flux tubes cannot connect with the hot gas and the clouds are insulated against destruction by thermal conduction.

In my discussion of quasar clouds for this meeting, I have emphasized issues regarding radiation hydrodynamics rather than astrophysical problems such as the question of cloud origin, but I did not wish to imply that these astrophysical problems are uninteresting or easily solved! Any cloud model requires a cloud production rate comparable to the flow time of the clouds through or across the quasar atmosphere. A variety of ideas have been proposed for cloud creation - mass loss from stars near the central black hole by ablation or other means, thermal instabilities in the hot cloud-confining gas, ejection of clouds from the surface of an accretion disk, etc. - but none of these suggestions is fully convincing or free from critisism.

Lawrence Anderson: Can you provide arguments against stellar ablation? It would seem that continuous formation as cometary tails from stars overcomes many of the confinement, variety of optical depths, and variety of formation radii problems. 
Mathews: I share your enthusiasm for this process, but the stars may not be as generous with their mass as we would like. A very rough estimate of the mass flux rate through the broad line region in quasars, $\sim 1 M_{\odot} y r^{-1}$, exceeds by several orders of magnitude the rate of stellar mass loss for a relatively old population of (non-colliding) stars within the central parsec. The mass ejected from normally evolving giant stars is insufficient. Unless many massive stars are continuously forming in this region, which would seem to be very unlikely indeed, the evolution of giant stars is not efficient enough for the mass flux required.

Reuven Opher: The conditions that you are talking about are ripe for a thermal instability for the formation of the line emitting clouds. Can you comment on the possibility of the thermal instability as the origin of the clouds?

Mathews: Thermal instabilities in a hot gas as a means of producing clouds have been studied by EILEK and CAROFF [45]. I have the impression that in general these processes are rather inefficient and that the preinstability physical situation has to be carefully (and somewhat artificially) prepared in order to make the instability occur as fast as one cloud flow time across the broad line region - such as for example considering a dominant pressure of relativistic electrons which are just about to radiate a large fraction of their energy by some mechanism. In addition, clumps formed by thermal instability are likely to be totally disrupted as soon as the first bit of gas cools to $\sim 10^{4} \mathrm{~K}$, at which time strong nonisotropic radiative forces must rapidly push the cool condensations through the rest of the incipient slowly cooling cloud. This differential acceleration would probably defocus and disrupt the coherence of the thermal instability mechanism.

Michel: Returning to the question of stellar ablation as a source of "clouds," why couldn't any star be ablated on passing close to the "central engine" simply by being smothered and unable to radiate, hence losing energy by mass loss instead?

Mathews: I have looked at the problem of driving gas off of dwarf main sequence (or arrested pre-main sequence) stars within the broad line region. In the solar neighborhood dwarf stars with masses near $0.1 M_{\odot}$ contain the bulk of stellar matter. The radiative energy received by these low mass stars in the broad line region from the quasar exceeds their own luminosities. This led me to speculate (MATHEWS [46]) that the additional internal circulation generated by radiative acceleration of gas in the stellar atmospheres could help drive energy and entropy deep into envelopes of these stars. However, there is some evidence from binary stars containing low mass dwarf stars and $x$-ray emitting compact objects that such a process is not working in that environment (VERBUNT, FABIAN, and REES [47]).

Vincent Icke: I'm a bit dissappointed that your'e leaving all hydrodynamics out. To mention but one problem, you're not at liberty to specify the surface of the clouds at you have done. Of course the flow problems are horrendous, but could you say if there are any observational constraints on the shapes and internal velocities of the clouds?

Mathews: I do not apoligize for the approach I have taken here! My own view, as one who has done a considerable amount of numerical hydrodynamics, is that progress on the problem of radiation hydrodynamics in quasar clouds is not easily made at the outset by attempting specific multidimensional numerical hydrodynamic calculations. To mention one of several numerical difficulties that come up when this approach is taken, I note that during the full evolution of a (successful) quasar cloud moving in the broad line region, it must traverse $R / r \sim 10^{6}$ cloud diameters. This could soak up an enormous amount of computer time, most of it spent in computing the flow in the hot intercloud medium, where the Courant limitation can be troublesome.

I am aware that the boundary conditions I have imposed on the clouds are approximate, 
but I believe they are be plausible and sufficiently accurate to discuss the issues I have raised. The semi-quantitative treatment $I$ have presented is sufficient to describe why velocity fields and cloud deformations should be expected, but is obviously inadequate to describe the details of this time-dependent process. To some extent, too, it is unnecessary to have available exact and detailed geometrical information about the clouds - either those in quasars or those in the daily sky - to understand their fundamental nature. There are at present no strong observational constraints on the shapes or internal velocity fields in the clouds; all of this information is hopelessly unresolved by any present telescope.

Miguel Ibanez: Which method do you use to study the stability problem? Do you linearize the equations? Do you use variational techniques or just follow the perturbation by numerical codes?

Mathews: In the work of Krolik, Mathews, and Blumenthal the method of linearization has been used. This approach is algebraically tedious and the results are usually obvious, at least in retrospect. For this reason, I have adopted a more heuristic and intuitive approach here, based on a simplification of the dispersion relation that results from the linear perturbation theory.

Mike Shull: You've only discussed Ly $\alpha$ as an optically [thick] line which develops substantial pressure. What about Mg II and C IV [lines], which would [be] less thick and therefore potentially more destabilizing?

Mathews: FERLAND and ELITZUR [48] have recently studied the dynamical importance of the MgII and CIV resonance lines. They argue that these lines could be dynamically disruptive if the densities of the clouds are too low or the ionization parameters much higher than the commonly accepted single-slab values.

L. Nobili: Just on theoretical grounds, we know that the high energy continuous emission occurring in the central regions of AGN's is highly anisotropic. On the other hand broad emission line clouds emit isotropically. We could therefore expect that the ratio of the continuum to the line emission depends strongly on the angle between the line of sight and the axis of the disc. Is this correct? Is there any observational evidence of this effect?

Mathews: Recently Netzer has offered an explanation of the Baldwin Effect using an argument similar to the one you have proposed. The Baldwin Effect is the empirical result that, for quasars having flat radio spectra, the equivalent width of the C IV $\lambda 1500$ line decreases with increasing quasar luminosity. Netzer can account for this by assuming that the ultraviolet continuua arise from accretion disks that are randomly oriented among the observed quasars, with the brighter ones seen pole-on.

K. P. Singh: The covering factor has been found to be variable in some sources. If it is due to movements of clouds or their destruction/creation, then what kind of variability amplitude and variability time scales can be expected?

Mathews: One expected time scale would be the dynamical time for a cloud to cross the broad line region, and the observed time scales of the absorption variation are consistent with this. Little can be predicted at this point regarding the variability amplitude.

D. Narasimha: At a temperature of $\sim 10^{10} \mathrm{~K}$ will the line broadening due to electron scattering in these clouds be appreciable?

Mathews: Usually the parameters of the hot intercloud medium can be arranged so that the optical depth to electron scattering of the broad line emission is considerably less than unity. Well-observed emission line profiles often have detailed structure superimposed on a fairly logarithmic shape. This structure, as well as the log-like profiles, are inconsistent with 
a model in which electron scattering is dominant. For one quasar, however, SHIELDS and MCKEE [49] have suggested that the very broad component to the emission profiles is due to electron scattering in the intercloud medium. 